\title{
Induction of Apoptosis by Ethyl Acetate Fraction of Astragalus membranaceus in Human Non-small Cell Lung Cancer Cells
}

\section{- Apoptosis Induction by Astragalus membranaceus -}

\author{
Hyun-Ji Park', Shin-Hyung Park ${ }^{1 *}$ \\ ${ }^{1}$ Departments of Pathology, College of Korean Medicine, Dong-eui University, Busan, Korea
}

\author{
Key Words \\ Astragalus membranaceus, non-small cell lung cancer, \\ apoptosis
}

\begin{abstract}
Objectives: The purpose of this study is to investigate the anti-cancer effects of different fractions of Astragalus membranaceus (AM) in human non-small cell lung cancer (NSCLC) cells.

Methods: We isolated hexane, ethyl acetate, and butanol fractions from crude ethanol extract of AM. The cell death was examined by MTT assay and trypan blue exclusion assay. Apoptosis was detected by DAPI staining, annexin V-PI double staining and cell cycle analysis. The expression of apoptosis-related proteins and mitogen-activated protein kinases (MAPKs) was examined by western blot.
\end{abstract}

Results: Among various fractions of AM, the ethyl acetate fraction of AM (EAM) showed the strongest cytotoxic effect in NSCLC cells. EAM reduced the cell proliferation in a time- and dose-dependent manner in NSCLC cells. In addition, EAM induced the chromatin

Received: Oct 13, 2018 Reviewed: Oct 16, 2018 Accepted: Nov 14, 2018

$(0$ This is an Open-Access article distributed under the terms of the Creative Common Attribution Non-Commercial License (http://creativecommons.org/licenses/by-nc/4.0/) which permits unrestricted noncommercial use, distribution, and reproduction in any medium, provided the original work is properly cited.

(2) This paper meets the requirements of KS X ISO 9706, ISO 9706-1994 and ANSI/NISO Z39.48-1992 (Permanence of Paper). condensation, and increased the population of sub-G1 phase and annexin V-positive cells in a time-dependent manner, indicating that EAM induced apoptosis in NSCLC cells. Consistently, EAM enhanced the expression of cleaved caspase- 8 and -9 , and induced the accumulation of cleaved- poly (ADP-ribose) polymerase (PARP). Among MAPK proteins, only ERK was dephosphorylated by EAM, suggesting that ERK might be related with EAM-induced apoptosis.

Conclusion: Our results clearly demonstrate that EAM exhibited anti-cancer effects in NSCLC cells by induction of apoptosis. We provide a valuable evidence which suggests that AM could be a desirable therapeutic option for treatment of NSCLC.

\section{Introduction}

Lung cancer is the leading cause of cancer-related death worldwide, and exhibits a poor 5-years survival rate less than $20 \%[1,2]$. Non-small cell lung cancer (NSCLC) accounts for about $80 \%$ of all lung cancers [1]. Cigarette smoking is a major risk factor along with other environmental and genetic risk factors for developing NSCLC [3]. Approximately $40 \%$ of newly diagnosed lung cancer patients are stage IV. For these patients, cytotoxic combination chemotherapy is the first-line therapy $[3,4]$. However, the response rate is limited and patients eventually develop resistance against

"Corresponding Author

Shin-Hyung Park. College of Korean Medicine, Dong-eui University, 52-57 YangJeong-ro, Busanjin-gu, Busan 47227, Korea.

Tel: +82-51-850-8646 Fax: +82-51-853-4036

E-mail: omdpark@deu.ac.kr 
chemotherapy, which contributes to the poor prognosis [5]. The median overall survival for patients challenged with chemotherapy is only approximately 8-10 months [3]. Thus, it is urgent to develop more effective therapeutic drugs to treat lung cancer.

Apoptosis is a well-known type of programmed cell death (PCD) initiated from a death receptor- or mitochondrial-mediated signals. These signals finally activate caspases and induce the accumulation of cleaved poly (ADP-ribose) polymerase (PARP), a cellular substrate of caspases. Apoptotic cells are morphologically characterized by membrane blebbing, chromatin condensation, and apoptotic body formation [6,7]. Because apoptosis plays a pivotal role in regulation of cancer growth and progression, induction of apoptosis in cancer cells has been a major mode of action of chemotherapeutic drugs [8].

Astragalus membranaceus(AM) called as Hwang gi in Korean has been traditionally used in Korea for medicinal purposes. It possesses a variety of activities including vital-energy tonifying, skin reinforcing, diuretic and tissue generative actions [9]. It also contains several types of bioactive compounds such as ploysaccharides, flavonoids, and saponins [10-13]. In recent years, AM extracts and its active compounds have been reported to exhibit anti-cancer effects in various cancers including gastric cancer, hepatocellular carcinoma, colorectal cancer, nasopharyngeal carcinoma, breast cancer, and lung cancer [14-18]. Especially, astragaloside IV, calycosin, and formonetin, components isolated from AM, suppressed the growth and metastasis of NSCLCs through regulation of immune system, induction of cell cycle arrest and apoptosis [19-24]. In addition, it is reported that AM increased the efficacy of platinum-based chemotherapy for patients with advanced NSCLC when based on syndrome differentiation [25]. Given that AM is known to mainly affect to the functions of lung according to the attributive channel theory, an oriental medicinal theory on the selective actions of herbal medicines on certain meridian channels and target organs [26], the current study investigated which fraction of AM exhibits the strongest anti-cancer activities in human NSCLC cells. Our results provide a basic information for finding further new anti-cancer components from the specific fraction of AM.

\section{Materials and Methods}

\subsection{Preparation of various fractions from $A M$}

Dried roots of AM were bought from Nuri Herb Co. Ltd. (YoungCheon, Gyeongsangbuk-do, Republic of Korea). AM (200 g) was chopped and extracted for three times with $1.5 \mathrm{~L}$ of $80 \%$ ethanol for 24 hours at room temperature with shaking (100 rpm) and occasional sonication. The extract was combined, filtered, and concentrated with a vacuum rotary evaporator (Yamato Scientific Co. Ltd., Tokyo, Japan) under reduced pressure and lyophilized. The powder was suspended in distilled water and further fractionated with three different solvents in a stepwise manner. The fractions were the hexane fraction (hexane fraction of AM,
HAM), ethyl acetate fraction (ethyl acetate fraction or AM, EAM), and butanol fraction (butanol fraction of AM, BAM). The ethanol, hexane, ethyl acetate, and butanol were all purchased from SK chemicals (Seoul, Republic of Korea). The extract powder was dissolved in dimethyl sulfoxide (DMSO; Amresco, Solon, OH, USA) as a stock solution at a $200 \mathrm{mg} / \mathrm{ml}$ concentration, and diluted with medium to the desired concentration prior to use.

\subsection{Cell culture}

NCI-H1299, NCI-H460 and A549 human lung carcinoma cell lines were purchased from the American Type Culture Collection (ATCC; Manassas, VA, USA). H1975 cells were kindly provided by professor Ho-Young Lee (Seoul national university, Seoul, Republic of Korea). Cells were cultured at $37^{\circ} \mathrm{C}$ in a humidified incubator under $5 \% \mathrm{CO}_{2}$ in RPMI 1640 (WelGENE, Daegu, Republic of Korea) supplemented with $10 \%$ fetal bovine serum (FBS, WelGENE) and $1 \%$ antibiotics (WelGENE).

\subsection{Chemicals, reagents and antibodies}

MTT[3-(4,5-dimethylthiazol-2-yl)-2,5-diphenyltetrazolium bromide] and trypan blue were bought from Duchefa (Haarlem, The Netherlands) and WelGENE, respectively. 4,6-dianmidino-2-phenylindole (DAPI), paraformaldehyde, and propidium iodide (PI) were obtained from Sigma-Aldrich (Sigma; St. Louis, MO, USA). Primary antibodies against phospho-ERK, phospho-p38, and phospho-JNK were obtained from Cell Signaling Technology (Beverly, MA, USA), and the other primary antibodies were purchased from Santa Cruz Biotechnology (Santa Cruz, Dallas, TX, USA). Anti-rabbit secondary antibody and anti-mouse secondary antibody were purchased from Enzo Life Sciences (Farmingdale, NY, USA) and Bethyl Laboratories (Montgomery, TX, USA), respectively. Unless otherwise stated, all other chemicals were purchased from Sigma.

\subsection{MTT assay}

For investigation of cell viability, $3 \times 10^{3}$ cells were seeded onto 96-well plates and treated with different fractions of AM for 72 hours. The MTT solution was added to the media at a final concentration of $0.4 \mathrm{mg} / \mathrm{ml}$ and incubated for 4 hours. The media were aspirated and $100 \mu \mathrm{l}$ of DMSO were added to each well to dissolve the formazan. The absorbance was measured at $540 \mathrm{~nm}$ using a microplate reader (SpectraMax M3; Molecular Devices, Sunnyvale, CA, USA).

\subsection{Trypan blue exclusion assay}

$2 \times 10^{4}$ cells were seeded in 12 -well plates and treated with EAM for various time periods. Then cells were collected and stained by trypan blue solution at a final concentration of $0.1 \%$. As dead cells are stained as blue color, the number of viable cells was calculated by counting the unstained cells using a hemocytometer. 


\subsection{Nuclear staining with DAPI}

$3 \times 10^{5}$ cells were seeded in 6 -well plates and treated with EAM for 72 hours. Then cells were collected, washed with cold phosphate-buffered saline (PBS), and fixed with $3.7 \%$ paraformaldehyde for 30 minutes at room temperature. The fixed cells were washed three times with cold PBS, and attached to slide glasses using a cytospin (Shandon, Pittsburgh, PA,USA). After staining with a DAPI solution $(2.5 \mu \mathrm{g} / \mathrm{ml})$ for 20 minutes at room temperature in the dark, cells were washed twice with PBS, and mounted with aqueous mounting medium (crystal/mount; Biomeda, Foster City, CA, USA). The nucleus was observed using a fluorescence microscope (Carl Zeiss, AG, Germany) at $\times 200$ magnification.

\subsection{Annexin V-PI double staining}

$1 \times 10^{5}$ cells were seeded in 6 -well plates and challenged with EAM for 72 hours. Then cells were harvested and double-stained with annexin V-FITC and PI using Annexin V-FITC Apoptosis Detection Kit I (BD Biosciences PharMingen, San Diego, CA, USA) according to the manufacturer's instructions. Annexin V-positive apoptotic cells were determined using a flow cytometer (FACS Caliber; Becton Dickinson, San Jose, CA, USA) and CellQuest software.

\subsection{Measurement of sub-G1 phase}

$1 \times 10^{5}$ cells were seeded in 6 -well plates and challenged with EAM for 72 hours. The cells were collected, washed with cold PBS, and fixed with cold $80 \%$ ethanol for 1 hour at $4^{\circ} \mathrm{C}$. After centrifugation, cells were stained with PI (final concentration, $50 \mu \mathrm{g} / \mathrm{ml}$ ) in the presence of $30 \mu \mathrm{g} / \mathrm{ml}$ DNase-free RNase A (Sigma) for 30 minutes in the dark at room temperature. The relative DNA content in each phase of the cell cycle was determined using a flow cytometer and CellQuest software.

\subsection{Western blot}

EAM-treated cells were lysed with cold radio-immunoprecipitation assay (RIPA) buffer (Thermo Scientific, Rockford, IL, USA) supplemented with a protease inhibitor cocktail (Thermo Scientific) and phosphatase inhibitors ( $1 \mathrm{mM} \mathrm{Na}_{3} \mathrm{VO}_{4}$ and $100 \mathrm{mM} \mathrm{NaF}$ ) for 1 hour on ice. After centrifugation at $13,000 \mathrm{rpm}$ at $4^{\circ} \mathrm{C}$ for 30 minutes, the supernatants were collected and protein concentration was determined using a biochinconinic acid (BCA) protein assay kit (Pierce Biotechnology, Rockford, IL, USA) according to the manufacturer's instruction. The same amounts of protein were resolved by sodium dodecyl sulfate (SDS)-polyacrylamide gels and transferred onto a polyvinyl difluoride (PVDF) membrane (Merck Millipore, Billerica, MA, USA). Then the membrane was blocked with 3\% bovine serum albumin (BSA; GenDEPOT, Barker, TX, USA) in TBST [Tris-buffered saline (TBS) containing $0.1 \%$ Tween 20 (Duchefa)] for 1 hour at room temperature, and incubated overnight with primary antibody at $4^{\circ} \mathrm{C}$. Follow- ing washing several times with TBST, the membrane was incubated with the secondary antibody for 1 hour at room temperature, and washed again with TBST multiple times. The protein expression was detected by SuperSignal West Pico Chemiluminescent Substrate (Thermo Scientific) according to the manufacturer's recommended protocol.

\subsection{Statistical analysis}

Each result is expressed as the mean $\pm \mathrm{SD}$ of data obtained from triplicate experiments. A statistical analysis was performed by Student's t-test. Differences less than $P<0.05$ were considered statistically significant.

\section{Results}

\subsection{Effects of different fractions of AM on cell sur- vival in NSCLC cells}

To investigate which fraction of AM exhibits the strongest anti-cancer effects in NSCLC cells, we performed MTT assay. Compared with the other fractions, ethyl acetate fraction of AM (EAM) exerted the strongest cytotoxicity in various NSCLC cell lines, including H1299, H460, A549 and H1975 cells. Although the hexane fraction of AM (HAM) also reduced the cell viability in a concentration-dependent manner in those cell lines, and the butanol fraction of AM (BAM) decreased the cell viability in H460 and H1975 cells, EAM generally exhibited more superb anti-cancer effects than HAM or BAM. BAM showed no cytotoxicity in H1299 and A549 cells (Figure 1A-D).

\subsection{Effects of EAM on cell proliferation in NSCLC cells}

We next investigated whether EAM suppresses cell proliferation in NSCLC cells using trypan blue exclusion assay. EAM treatment markedly inhibited the cell proliferation in H1299, A549, H460, and H1975 cells in a time- and concentration-dependent manner (Figure 2A-D). The proliferation-inhibitory effect was more significant in H1299 and H1975 cells than in H460 and A549 cells. These results clearly indicate that EAM exhibits anti-cancer effects through suppression of cell proliferation in NSCLC cells.

\subsection{Effects of EAM on apoptosis induction in NS- CLC cells}

To determine whether the anti-proliferative effects of EAM was due to apoptosis induction, we performed annexin V-PI double staining assay. As shown in Figure 3A and $3 \mathrm{~B}$, the rate of annexin $\mathrm{V}$-positive apoptotic cells was significantly increased by EAM treatment in both H1299 and A549 cells (Figure 3A and 3B). Similar results were obtained when apoptosis was monitored by flow cytometry cell cycle analysis. EAM treatment enhanced the sub-G1 phase cell population which means apoptotic cells in a 
A)

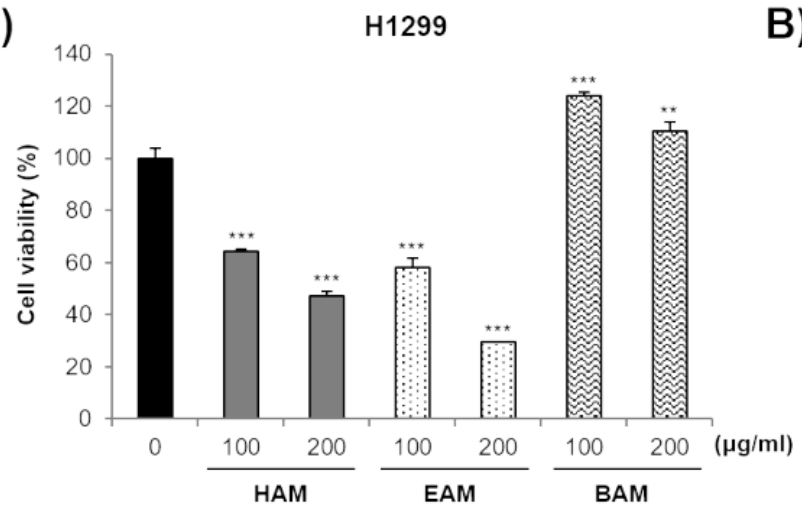

C)

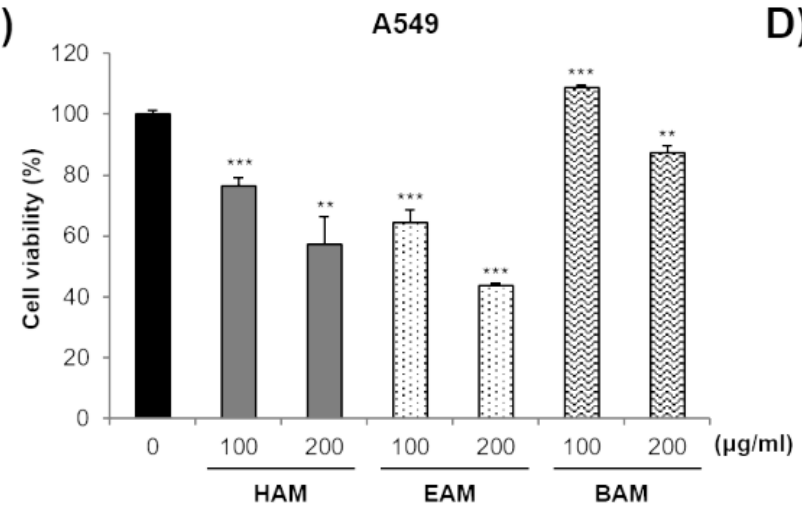

B)

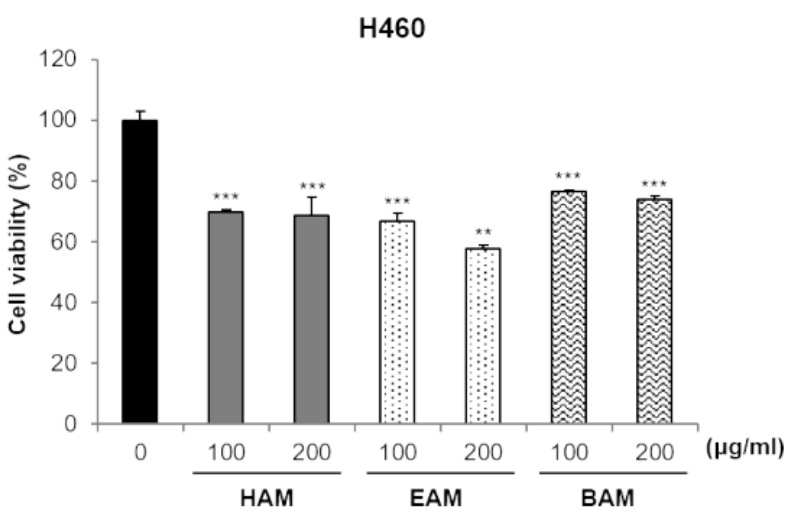

D)

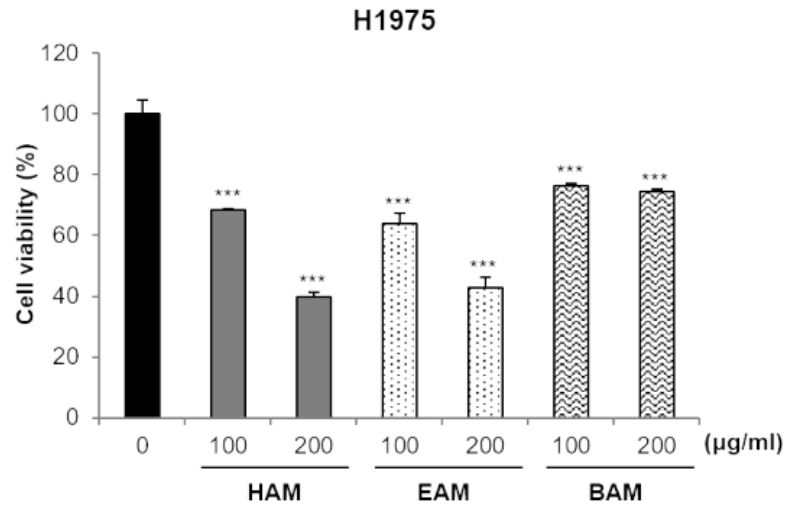

Figure 1 Effects of the different fractions of AM on cell survival in NSCLC cells.

H1299 (A), H460 (B), A549 (C), and H1975 (D) human NSCLC cells were seeded onto 96 well plates and treated with the different fractions of AM for $72 \mathrm{~h}$. The cell viability was evaluated by MTT assay. Data are expressed as the mean \pm S.D. of three independent experiments. Significance was determined by the Student's t-test ${ }^{* *} P<0.01,{ }^{* * *} P<0.001$ vs. untreated control). 
A)

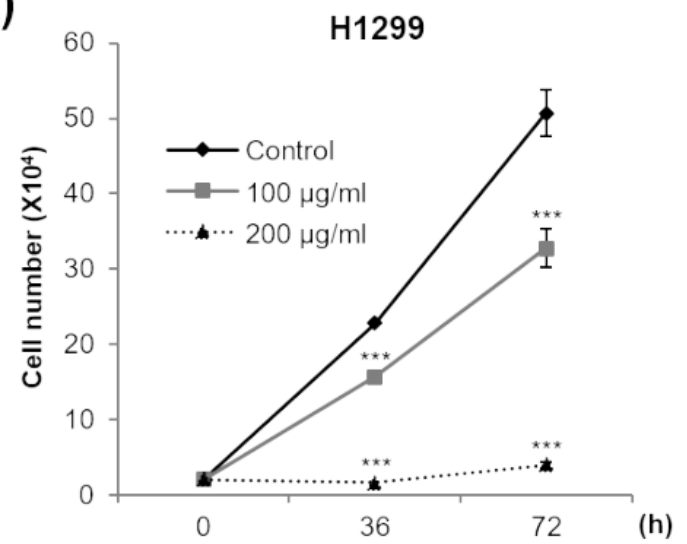

C)

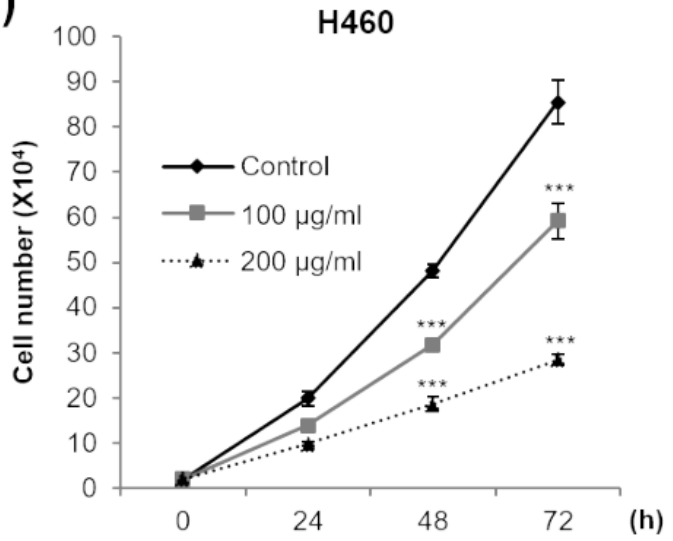

B)

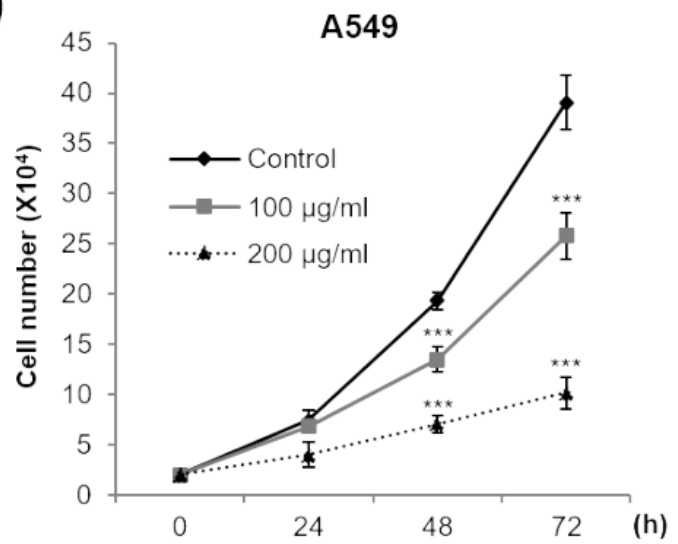

D)

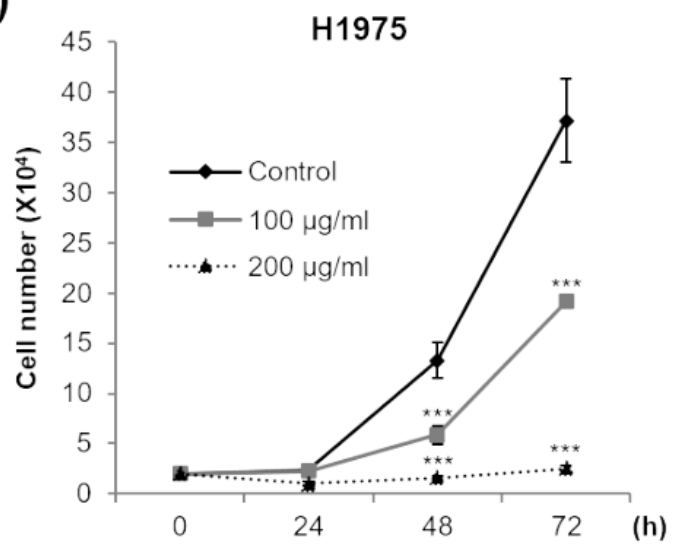

Figure 2 Effects of AM on cell proliferation in NSCLC cells.

H1299 (A), A549 (B), H460 (C), and H1975 (D) human NSCLC cells were seeded onto 12 well plates and treated with the indicated concentration of EAM for various time periods. The viable cell was evaluated by trypan blue exclusion assay. Data are expressed as the mean \pm S.D. of three independent experiments. Significance was determined by the Student's t-test ${ }^{* * *} P<0.001$ vs. untreated control). 
A)

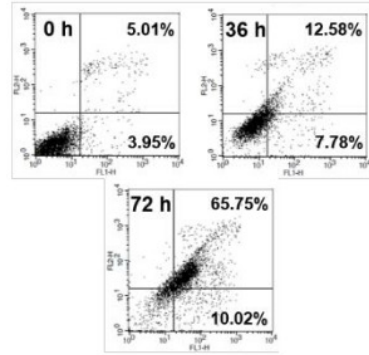

C)

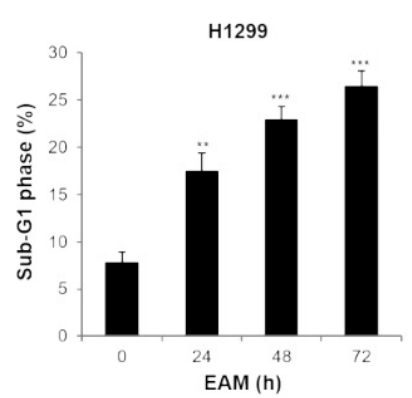

B)

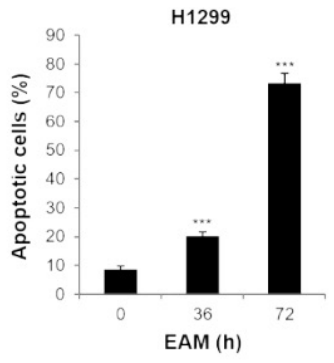

D)

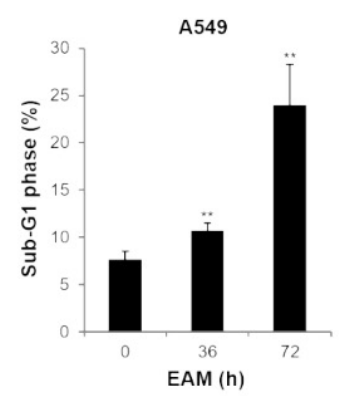

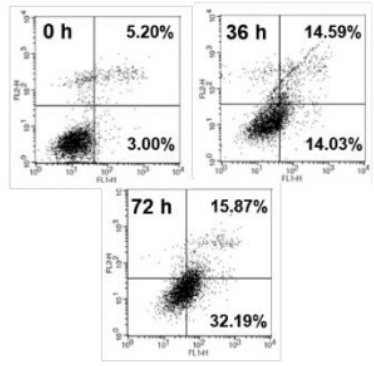

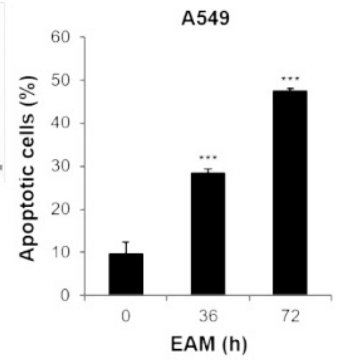

E)

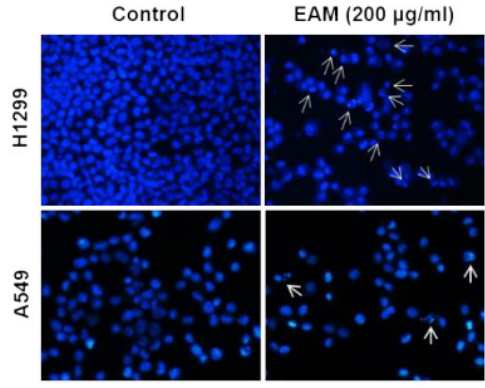

Figure 3 Effects of EAM on apoptosis induction in NSCLC cells.

(A-D) H1299 (A and C) and A549 (B and D) cells were seeded onto 6 well plates and treated with EAM (200 $\mu \mathrm{g} / \mathrm{ml})$ for indicated time periods. (A and B) Annexin V/PI double staining assay was conducted using a flow cytometer. Annexin V-positive population was determined as apoptotic cells. (C and D) Cells were stained with PI solution and sub-G1 DNA content was evaluated using a flow cytometer. Data are expressed as the mean \pm S.D. of three independent experiments. Significance was determined by the Student's t-test ( ${ }^{* *} P<0.01$, ${ }^{* * *} P<0.001$ vs. untreated control). (E) H1299 (upper) and A549 (lower) cells were treated with EAM $(200 \mu \mathrm{g} / \mathrm{ml})$ for $72 \mathrm{~h}$. To observe the changes of nuclear morphology, cells were stained with DAPI solution. The stained nuclei were observed under fluorescent microscope using blue filter (original magnification, 400X). The apoptotic nuclei were indicated as white arrow.

A)

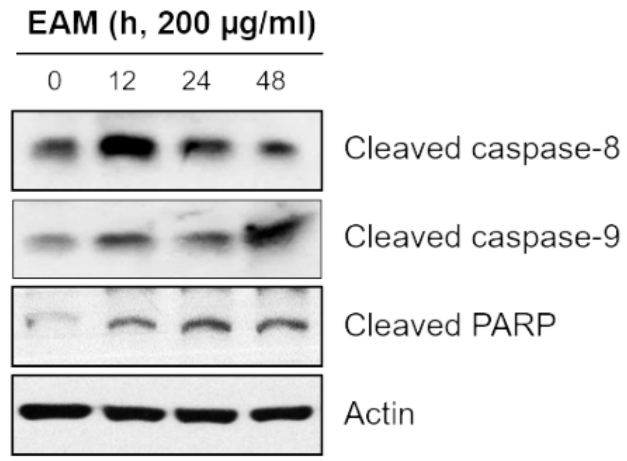

B)

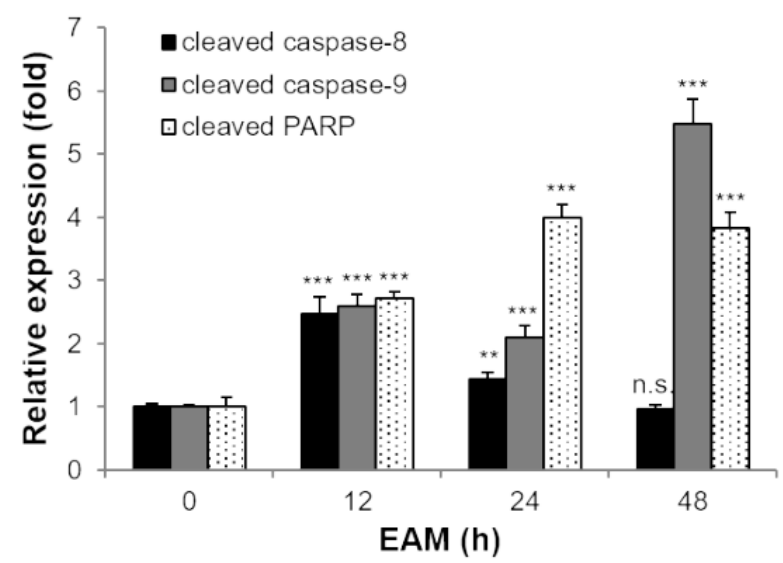

Figure 4 Effects of EAM on caspases activation in NSCLC cells.

(A) $\mathrm{H} 1299$ cells were treated with EAM $(200 \mu \mathrm{g} / \mathrm{ml})$ for indicated time periods. Cells were lysed and the cellular proteins were separated by SDS-polyacrylamide gels, transferred onto PVDF membrane. The membrane was probed by the indicated antibodies, and the proteins were visualized by an ECL detection system. Actin was used as an internal control. (B) The relative expression of cleaved caspase-3, -8, and PARP compared with untreated control was analyzed with Image J software using actin for normalization. Data are expressed as the mean \pm S.D. of three independent experiments. Significance was determined by the Student's t-test (n.s. Not significant, ${ }^{* *} P<0.01,{ }^{* * *} P<0.001$ vs. untreated control). 
A)

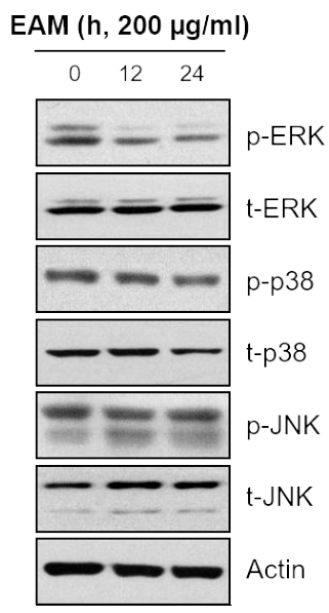

B)

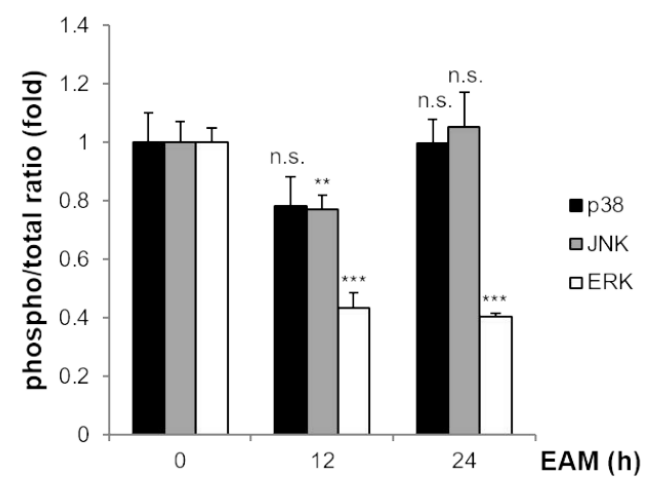

Figure 5 Effects of EAM on MAPK pathways in NSCLC cells.

(A) H1299 cells were treated with EAM $(200 \mu \mathrm{g} / \mathrm{ml})$ for indicated time periods. The cell lysates were subjected for western blot analysis. The expression levels of the indicated proteins were assessed. Actin was used as a loading control. (B) The ratio of p-ERK/t-ERK, p-p38/t-p38, and p-JNK/t-JNK was analyzed with Image J software using actin for normalization. Data are expressed as the mean \pm S.D. of three independent experiments. Significance was determined by the Student's t-test (n.s. Not significant, ${ }^{* *} P<0.01,{ }^{* * *} P<0.001$ vs. untreated control).

time-dependent manner in both H1299 and A549 cells (Figure 3C and 3D). Next, we investigated the morphological changes in nucleus to verify apoptosis induction following 72 hours treatment of EAM in NSCLC cells. As shown in Figure 3E, EAM-treated cells showed highly condensed and fragmented nuclei which indicate apoptotic cells in both H1299 and A549 cells (Figure 3E). Taken together, these results demonstrate that EAM treatment induced apoptotic cell death in NSCLC cells.

\subsection{Effects of EAM on caspase activation in NS- CLC cells}

To determine whether caspase activation is involved in EAM-induced apoptosis, we next conducted western blot analysis. Apoptotic caspases are activated upon the receipt of either an extrinsic or an intrinsic death signal, leading to a proteolytic cascade which amplifies the apoptotic signaling pathway. The signals from the extrinsic and intrinsic pathways activate caspase-8 and -9, respectively [27]. Our results showed that the expression of cleaved caspase- 8 and -9 was commonly increased by EAM treatment in H1299 cells, suggesting that EAM activated both extrinsic and intrinsic apoptotic pathways (Figure 4A and 4B). Notably, the expression of cleaved capsase- 8 and -9 increased the most at 12 hours and 48 hours posttreatment of EAM, respectively. These results suggest that EAM induced apoptosis at early time through the extrinsic pathway and at late time through the intrinsic pathway. In addition, the expression of cleaved PARP, an apoptosis marker protein which is cleaved by caspases and leads to the degradation of nuclear materials and apoptotic morphological modifications, was up-regulated by EAM treatment (Figure 4A and 4B). Collectively, these results demonstrate that EAM induced apoptosis by activation of caspases.

\subsection{Effects of EAM on the MAPK pathway in NS- CLC cells}

To explore the molecular mechanism of EAM-induced apoptosis, we next investigated the influence of EAM on the activation of MAPK proteins. In response to a wide range of stimuli, distinct MAPK pathways exert a variety of cellular outputs, such as cell proliferation, cell cycle arrest, migration, and apoptosis [28]. Among three family members of MAPKs including ERK, p38 and JNK, EAM treatment markedly suppressed the phosphorylation of ERK in a time-dependent manner while it had no significant effect on the expression of phospho-JNK and phospho-p38 in H1299 cells (Figure 5A and 5B). These results suggest that ERK might be involved in EAM-induced apoptosis.

\section{Discussion}

The present study explored the anti-cancer effects of various fractions of AM in human NSCLC cells. We found that the ethyl acetate fraction of AM exhibited the strongest inhibitory effects on the cell survival and proliferation in NSCLC cell lines with different genetic backgrounds. EAM treatment induced apoptosis proved by chromatin condensation, accumulation of sub-G1 phage cells and annexin V-positive cells. EAM also induced the activation of caspases and subsequent cleavage of PARP. These results collectively demonstrate that EAM exhibits anti-cancer activities by apoptosis induction in NSCLC cells.

The extrinsic pathway is known as a death-receptor pathway. It is initiated by the interactions between the death 
ligands and their corresponding receptors including Fas ligand/Fas, tumor necrosis factor (TNF)- $\alpha / T N F$ receptor, and TNF-related apoptosis-inducing ligand (TRAIL)/ TRAIL receptor. They form the death-induced signaling complex (DISC) to activate caspase-8. Sometimes the extrinsic pathway is connected with the intrinsic pathway by the cleavage of Bid mediated by caspase- 8 . The truncated Bid permeabilizes the mitochondrial outer membrane and inhibits the function of mitochondria, which initiates the intrinsic pathway, like other pro-apoptotic proteins including Bim, Bad, and Bax. The release of cytochrome c from the mitochondrial intermembrane space finally activates caspase-9 $[27,29]$. Our results showed that the cleavage of caspase- 8 occurred earlier $(12 \mathrm{~h}$ posttreatment of EAM) than that of caspase-9 (48 hours posttreatment of EAM), suggesting that EAM activated the extrinsic pathway at early time, but the intrinsic pathway at late time. The cleavage of Bid might have mediated this process.

Although several single compounds of AM, including astragaloside IV, calycosin, and formonetin, were proved to possess anti-cancer effects in NSCLC [19-24], it is still needed to find more effective compounds from AM to understand the whole mechanism of anti-cancer effects of AM. In this respect, the current study provides a basic information for finding further new anti-cancer components from the specific fraction of AM. Because oriental herbs generally possess multiple mechanisms of action, it can be an interesting next topic to explore whether the active compounds isolated from the ethyl acetate fraction of AM exhibits synergistic anti-cancer effects in NSCLC.

The current study did not determine the precise molecular mechanism of EAM-induced apoptosis. However, we showed that EAM suppressed the phosphorylation of ERK, but not JNK and p38, which suggests that inhibition of ERK might contribute to the apoptosis induction. ERK is an important subfamily of MAPK that regulate a broad range of cellular activities and physiological processes. Generally, activation of ERK promotes cell survival even though it can exhibit pro-apoptotic functions under certain conditions [30]. In our case, it seems that ERK acted as a survival factor to resist to apoptotic cell death. Several studies have reported that inhibition of ERK pathway triggered apoptosis in NSCLC cells [31-33]. Juhnn and Kim reported that cAMP signaling augmented radiation-induced apoptosis via inhibition of the Raf-MEK-ERK pathway in NSCLC cells [31]. Zhou et al. demonstrated that blocking PI3K/AKT and MEK/ERK pathways overcome gefitinib-resistance in NSCLC cells [32]. Deng et al. also reported that melatonin enhanced the anticancer effect of berberine by inhibition of several pathways including ERK pathway in NSCLC cells [33]. These reports consistently support our hypothesis that EAM induced apoptosis by blocking ERK pathway in NSCLC cells.

\section{Conclusion}

In conclusion, our study demonstrated that EAM had the strongest anticancer activities among various fractions of AM by induction of apoptosis in NSCLC cells. We provide a valuable evidence which suggests that AM could be a de- sirable therapeutic option for treatment of NSCLC. Further studies are warranted to investigate the specific target of EAM and evaluate the effectiveness of EAM in additional preclinical and clinical settings.

\section{Acknowledgment}

This study was supported by grants from the National Research Foundation of Korea (NRF), Republic of Korea (No. NRF-2016R1C1B2015076), and by grants from the Dongeui University Grant (No. 201802780001), Republic of Korea.

\section{Conflict of interest}

The authors declare that there are no conflicts of interest.

\section{References}

1. Visbal AL, Williams BA, Nichols FC 3rd, Marks RS, Jett JR, Aubry MC, et al. Gender differences in non-smallcell lung cancer survival: an analysis of 4618 patients diagnosed between 1997 and 2002. Ann Thorac Surg. 2004;78:209-15.

2. Keith R, Miller Y. Lung cancer chemoprevention: current status and future prospects. Nat Rev Clin Oncol. 2013;10:334-43.

3. Zappa C, Mousa SA. Non-small cell lung cancer: current treatment and future advances. Transl Lung Cancer Res. 2016;5(3):288-300.

4. Ramalingam S, Belani C. Systemic chemotherapy for advanced non-small cell lung cancer: recent advances and future directions. Oncologist. 2008;Suppl 1:S5-13.

5. Blackhall FH, Shepherd FA, Albain KS. Improving survival and reducing toxicity with chemotherapy in advanced nonsmall cell lung cancer: a realistic goal? Treat Respir Med. 2005;4:71-84.

6. Wolf BB, Green DR. Suicidal tendencies: apoptotic cell death by caspase family proteinases. Journal of Biological Chemistry. 1999;274:20049-52.

7. Susin SA, Daugas E, Ravagnan L, Samejima K, Zamzami N, Loeffler M, et al. Two distinct pathways leading to nuclear apoptosis. Journal of Experimental Medicine. 2000;192:571-80.

8. Makin G, Hickman JA. Apoptosis and cancer chemotherapy. Cell Tissue Res. 2000;301(1):143-52.

9. Auyeung KK, Han QB, Ko JK. Astragalus membranaceus: a review of its protection against inflammation and gastrointestinal cancers. Am J Chin Med. 2016;44(1):1-22.

10. Ma Y, Liu C, Qu D, Chen Y, Huang M, Liu Y. Antibacterial evaluation of sliver nanoparticles synthesized by polysaccharides from Astragalus membranaceus roots. Biomed Pharmacother. 2017;89:351-57.

11. Zhu J, Zhang H, Zhu Z, Zhang Q, Ma X, Cui Z, et al. Effects and mechanism of flavonoids from Astragalus complanatus on breast cancer growth. Naunyn Schmiedeberg's Arch Pharmacol. 2015;388(9):965-72.

12. Wang Y, Auyeung KK, Zhang X, Ko JK. Astragalus saponins modulates colon cancer development by regu- 
lating calpain-mediated glucose-regulated protein expression. BMC Complement Altern Med. 2014;14:401.

13. Denzler K, Moore J, Harrington H, Morrill K, Huynh $\mathrm{T}$, Jacobs B, et al. Characterization of the physiological response following in vivo administration of Astragalus membranaceus. Evidence-based complementary and alternative medicine. 2016;6861078.

14. Wang Z, Dong L, Zhen Y, Wang Y, Qi D, Xu A, et al. Astragalus extract inhibits proliferation but enhances apoptosis in gastric cancer. BMC Complement Altern Med. 2016;29(5):1473-82.

15. Huang WH, Liao WR, Sun RX. Astragalus polysaccharide induces the apoptosis of human hepatocellular carcinoma cells by decreasing the expression of Notch1. Int J Mol Med. 2016;38(2):551-57.

16. Tseng A, Yang $\mathrm{CH}$, Chen $\mathrm{CH}$, Chen $\mathrm{CH}$, Hsu SL, Lee $\mathrm{MH}$, et al. An in vivo molecular response analysis of colorectal cancer treated with Astragalus membranaceus extract. Oncol Rep. 2016;35(2):659-68.

17. Lai X, Xia W, Wei J, Ding X. Therapeutic effect of Astragalus polysaccharides on hepatocellular carcinoma H22-bearing mice. Dose-response. 2017;15(1):1-6.

18. Zhou Z, Meng M, Ni H. Chemosensitizing effect of Astragalus polysaccharides on nasopharyngeal carcinoma cells by inducing apoptosis and modulating expression of Bax/Bcl-2 ratio and caspases. Medical science monitor: international medical journal of experimental and clinical research. 2017;23:462-69.

19. Cheng XD, Gu JF, Yuan JR, Feng L, Jia XB. Suppression of A549 cell proliferation and metastasis by calycosin via inhibition of the PKCalpha/ERK1/2 pathway: an in vitro investigation. Mol Med Rep. 2015;12(6):79928002.

20. Jia L, Lv D, Zhang S, Wang Z, Zhou B. Astragaloside IV inhibits the progression of non-small cell lung cancer through Akt/GSK-3 $\beta / \beta$-catenin pathway. Oncol Res. 2018;21:10.

21. He CS, Liu YC, Xu ZP, Dai PC, Chen XW, Jin DH. Astragaloside IV Enhances Cisplatin Chemosensitivity in Non-Small Cell Lung Cancer Cells Through Inhibition of B7-H3. Cell Physiol Biochem. 2016;40(5):1221-9.

22. Yang Y, Zhao Y, Ai X, Cheng B, Lu S. Formononetin suppresses the proliferation of human non-small cell lung cancer through induction of cell cycle arrest and apoptosis. Int J Clin Exp Pathol. 2014;7(12):8453-61.

23. Zhang A, Zheng Y, Que Z, Zhang L, Lin S, Le V, et al. Astragaloside IV inhibits progression of lung cancer by mediating immune function of Tregs and CTLs by interfering with IDO. J Cancer Res Clin Oncol. 2014;140(11):1883-90.

24. Guo L, Bai SP, Zhao L, Wang XH. Astragalus polysaccharide injection integrated with vinorelbine and cisplatin for patients with advanced non-small cell lung cancer: effects on quality of life and survival. Med Oncol. 2012;29(3):1656-62.

25. Wang SF, Wang Q, Jiao LJ, Huang YL, Garfield D, Zhang J, et al. Astragalus-containing traditional Chinese medicine, with and without prescription based on syndrome differentiation, combined with chemotherapy for advanced non-small-cell lung cancer: a systemic review and meta-analysis. Current oncology.
2016;23(3):188-95.

26. Liu SQ. Clinical application of the theory of the attributive channel of traditional Chinese medicine. Zhong Yao Tong Bao. 1986;11:56-8.

27. Wolf BB, Green DR. Suicidal tendencies: apoptotic cell death by caspase family proteinases. J Biol Chem. 1999;274:20049-52.

28. Ouyang L, Shi Z, Zhao S, Wang FT, Zhou TT, Liu B, et al. Programmed cell death pathways in cancer: a review of apoptosis, autophagy and programmed necrosis. Cell Prolif. 2012;45(6):487-98.

29. Wada T, Penninger JM. Mitogen-activated protein kinases in apoptosis regulation. Oncogene. 2004;23(16):2838-49.

30. Lu Z, Xu S. ERK1/2 MAP kinases in cell survival and apoptosis. IUBMB Life. 2006;58(11):621-31.

31. Kim EJ, Juhnn YS. Cyclic AMP signaling reduces sirtuin 6 expression in non-small cell lung cancer cells by promoting ubiquitin-proteasomal degradation via inhibition of the Raf-MEK-ERK (Raf/mitogen-activated extracellular signal-regulated kinase/extracellular signal-regulated kinase) pathway. J Biol Chem. 2015;290(15):9604-13.

32. Li H, Schmid-Bindert G, Wang D, Zhao Y, Yang X, Su $\mathrm{B}$, et al. Blocking the PI3K/AKT and MEK/ERK signaling pathways can overcome gefitinib-resistance in non-small cell lung cancer cell lines. Adv Med Sci. 2011;56(2):275-84.

33. Lu JJ, Fu L, Tang Z, Zhang C, Qin L, Wang J, et al. Melatonin inhibits AP-2 $\beta /$ hTERT, NF-KB/COX-2 and Akt/ ERK and activates caspase/Cyto $C$ signaling to enhance the antitumor activity of berberine in lung cancer cells. Oncotarget. 2016;7(3):2985-3001. 